Wen Sheng, Zhang Tiemin*, Zhang Jiantao and Yang Xiuli

\title{
Optimal Design of Cymbal Stack Transducer in a Piezoelectric Linear Actuator by Finite Element Method
}

\begin{abstract}
The optimal design of a piezoelectric linear actuator using parametric optimum method-based finite element method (FEM) was presented. First, the FEM model of the cymbal stack transducer was generated with its initial configuration. The structural parameters were chosen as the design variables and the displacement on the top surface of the transducer taken as the objective function. Second, the zero-order optimization method was chosen as the basic tool of the structural updating. The structural optimization scheme of the cymbal stack transducer was carried out based on ANSYS parametric design language (APDL). Finally, an example of dynamic response analysis was performed on the cymbal stack transducer to verify the structural optimization scheme. The results show that the displacement on the top surface is increased by $32.9 \%$ compared with the case of initial configuration.
\end{abstract}

Keywords: cymbal stack transducer, piezoelectric linear actuator, finite element method, parametric optimum

DOI 10.1515/ehs-2015-0006

\section{Introduction}

Small piezoelectric actuators have been used in the fields of robot technology and information devices for their outstanding characters such as simple functional mechanism, compact structure, rapid response to external excitation and immunity from magnetic interference (Watson, Friend, and Yeo 2009; Chen and Liu 2013).

*Corresponding author: Zhang Tiemin, College of Engineering, South China Agricultural University, Guangzhou 510642, China, E-mail: tm-zhang@163.com

Wen Sheng: E-mail: vincen@scau.edu.cn, Zhang Jiantao: E-mail: tm-zhang@163.com, Yang Xiuli: E-mail: xylscau@163.com, College of Engineering, South China Agricultural University, Guangzhou 510642, China
Different types of piezoelectric actuators, such as inchworm ( $\mathrm{Lu}$ et al. 2009), traveling wave (Liu et al. 2010) and standing wave (Szufnarowski and Schneider 2011), have been developed and studied by numerous researchers. Although the existing actuators have exhibited excellent performance, there are still some problems to be overcome, for example, it is difficult for piezoelectric actuators to perform a linear driving system with a simple structure (Morita et al. 2012). In recent years, piezoelectric inertia actuator (PIA) has received a growing amount of attention to the fact that PIA has a simple construction and is controlled by a single signal, which allows for low production costs and simplifies miniaturization (Duan and Wang 2005; Lee et al. 2011). The PIA utilizes the inertia of a mass to drive a slider via friction contact. The principle behind the PIA is a stick-slip motion achieved by the piezoelectric effect (Hunstig, Hemsel, and Sextro 2013; Bajuri et al. 2012). Mostly, the displacement directly produced by piezoceramic cannot satisfy the need of the PIA (Chen et al. 2009). Therefore, people began to find a new way to amplify the displacement of the piezoceramic. The "Cymbal" transducer, which consists of a poled piezoelectric disk bonded to truncated conical metal endcaps, can produce 40 times displacement of the piezoceramic with the same dimension (Tsivgouli et al. 2007).

Recently, many researchers have paid increasing attention to evolution-based algorithms in the structural optimization field. These algorithms are robust and can provide a more reliable result to obtain the global optimum. What's more, they do not require continuity and derivative existence of the objective function. Genetic algorithms (GAs) are applied to composite structural optimization problems (Chakraborti 2004; Alibeigloo, Shakeri, and Morowa 2007; Ameri, Aghdam, and Shakeri 2012). Particle swarm optimization (PSO) is another evolutionary global algorithm, which has gained popularity (Poli, Lee, and Kwak 2007; Kulkarni and Venayagamoorthy 2011). In general, one of the main advantages of PSO over GA is its algorithmic simplicity. The PSO algorithm does not involve complicated 
encoding, decoding or special genetic operators, and thus it has fewer parameters to be adjusted. Although there has been much research concerning the optimization of piezoelectric structures (Araujo et al. 2013; Park, Lee, and Kwak 2012; Kim, Wang, and Brennan 2011; Jayachandran, Guedes, and Rodrigues 2011), few works aimed to optimizing the structure of the cymbal transducer.

In the present paper, a miniaturized piezoelectric linear actuator based on cymbal stack transducer is proposed here. An effective and automated design strategy is used to design the cymbal stack transducer. In this strategy, finite element analysis, parametrical model and a numerical optimization algorithm are integrated to create an automated design tool. Using this approach, shape design of the cymbal stack transducer is formulated in the form of an optimization problem that can be solved easily by a conventional numerical optimization algorithm.

\section{Description of Configuration and Driving Principle}

The stator of the piezoelectric linear actuator is composed of a shaft, a metal block and the cymbal stack transducer, as shown in Figure 1. The linear slider is preloaded on the shaft, which consists of two materials, as shown in Figure 2. The outer material is stainless steel for the basic inertial weight, and the inner material is resin with high friction factor, such as break material of automobile.

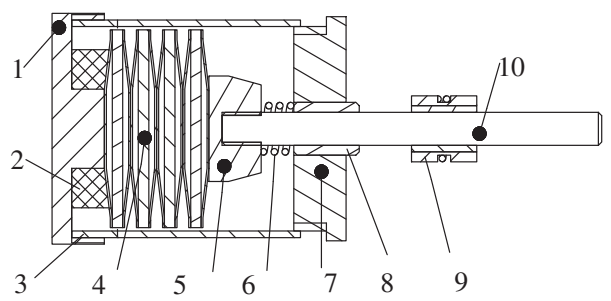

Figure 1: Schematic of the piezoelectric linear actuator. Note: 1, pedestal; 2, rubber pad; 3, sleeve; 4, cymbal stack transducer; 5 , metal block; 6 , spring; 7 , screw cap; 8 , linear bearing; 9 , linear slider; 10 , shaft.

The slider is driven by two successive motions, namely, slow forward movement and rapid backward movement. As shown in Figure 3, these motions may correspond to a sawtooth displacement. The driving procedure is as follows.

From (a) to (b): During the slow forward movement, the voltage signal is applied as shown in Figure 4. The cymbal stack transducer will produce extension

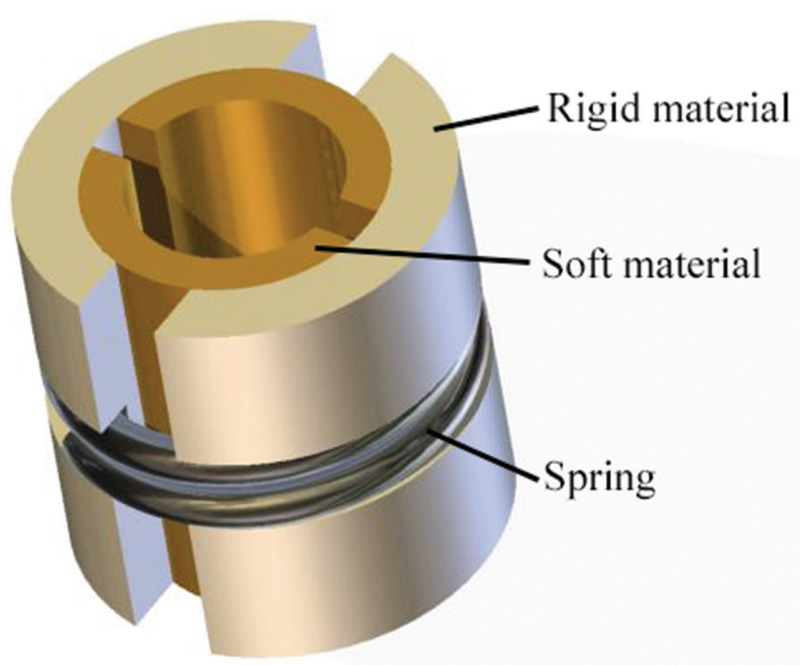

Figure 2: Structure of the slider.

(a)

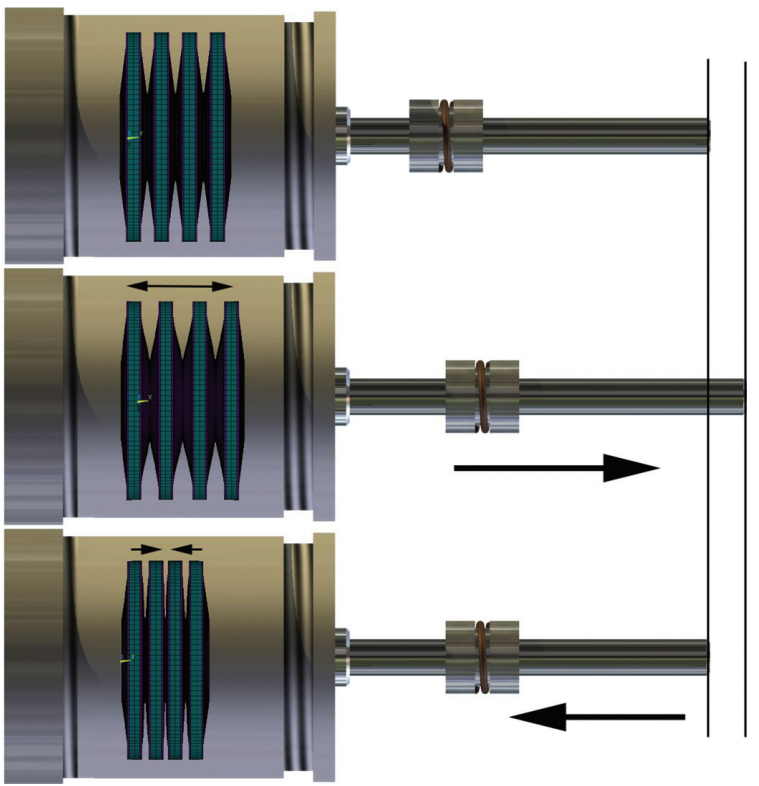

Figure 3: The principle of inertia displacement.

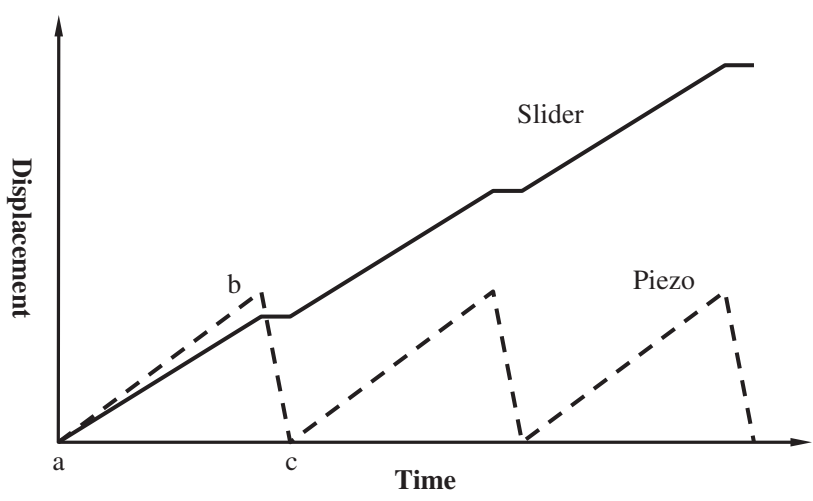

Figure 4: The sawtooth electrical potential. 
displacement in horizontal direction and push the shaft to move forward. The slider slowly moves with the stator by the frictional force. This movement is called stick motion.

From (b) to (c): Subsequently, when the shaft moves quickly backward to its original position, a slip will be produced between the linear slider and the shaft because the inertial force is larger than the static frictional force. Therefore, the linear slider remains at the location where it was placed during sticking motion.

By repeating these two stages, the slider can be driven a long distance. Reversible slider movement is possible by changing the order of slow and rapid movements of the piezoelectric linear actuator.

\section{Parameters Optimization Design}

The driving capability of the piezoelectric linear actuator depends on the energy conversion efficiency of the cymbal stack transducer. Because of small size, the structure parameters of the cymbal stack transducer have important influence on converting electric energy into mechanical energy. So, it is necessary to optimize the design of the cymbal stack transducer.

In this section, a novel design method, using finite element analysis and the zero-order optimization method, is proposed and applied to the design of the cymbal stack transducer. The finite element method (FEM) is applied to modal analysis and harmonic analysis of the cymbal stack transducer. The zero-order optimization method is used to locate optimum design of the design variables.

\section{Finite Element Model}

The aim of numerical modeling is to calculate natural frequencies and modal shapes of the cymbal stack transducer and to perform harmonic or transient response analysis. Basic equations for the motion of the cymbal stack can be written in matrix form (Narayanan and Schwartz 2007):

$$
\begin{gathered}
{[M] \frac{\partial^{2}\{u\}}{\partial^{2} t}+[C] \frac{\partial\{u\}}{\partial t}+\left[K_{1}\right]\{u\}+\left[K_{2}\right]\{\Phi\}=\left\{F_{N}\right\}} \\
{\left[K_{2}\right]^{T}\{u\}+\left[K_{3}\right]\{\Phi\}=\{Q\}}
\end{gathered}
$$

where $\{u\}$ is the nodal displacement vector, $\{\Phi\}$ is the electrical potential vector, $[M]$ is the mass matrix,
$[C]$ is the damping matrix, $\left[K_{1}\right]$ is the stiffness matrix, $\left[K_{2}\right]$ is the piezoelectric matrix, $\left[K_{3}\right]$ is the dielectric matrix, $\{Q\}$ is the nodal electrical charge vector and $\left\{F_{N}\right\}$ is the nodal mechanical force vector. The solution for nodal displacement is obtained with ANSYS. Figure 5 (a) shows the three-dimensional finite element model of the cymbal stack transducer. The endcaps material is brass, and the piezoelectric material is PZT-5A. The endcaps and piezoceramics were modeled with SOLID5 which is an element type in the library of ANSYS, defined by eight nodes each having three degrees of freedom. The complete model consists of 64,320 elements. The structural parameters needed for fabrication of a cymbal transducer are shown in Figure 5(b), where $t_{\mathrm{p}}$ and $t_{\mathrm{m}}$ are the thickness of piezoelectric disc and metal cap respectively, $t_{\mathrm{c}}$ is the height of the endcaps. $D, D_{1}$ and $D_{2}$ are the diameters of the piezoelectric disc, bottom and top of cavities, respectively. The initial values of the structural parameters are shown in Table 1.

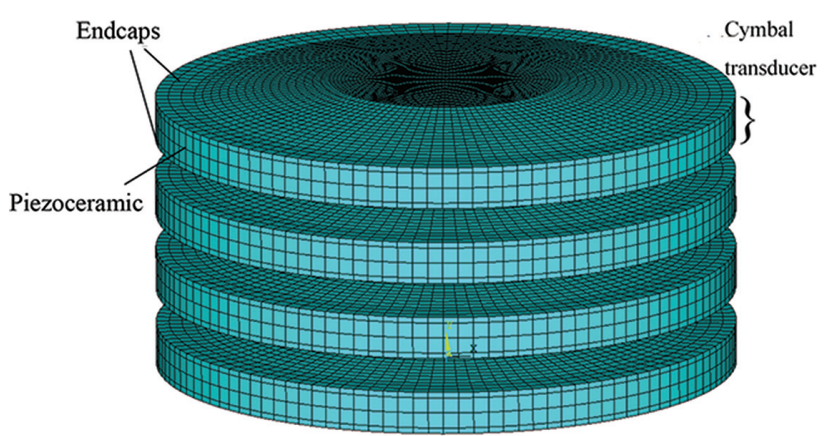

(a)

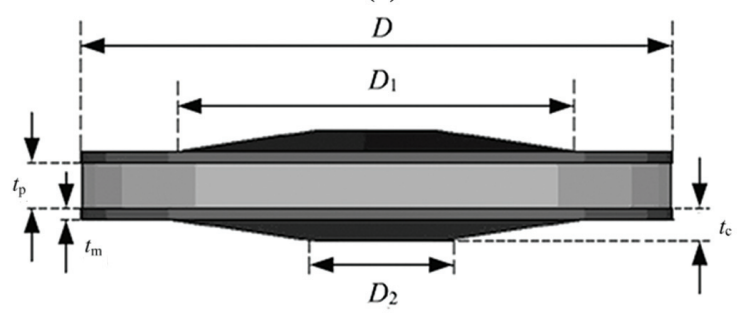

(b)

Figure 5: (a) Finite element model of the cymbal stack transducer. (b) Structure parameters of the cymbal transducer.

Table 1: The initial values of the structural parameters.

\begin{tabular}{lrrr}
\hline $\begin{array}{l}\text { Structural } \\
\text { parameter }\end{array}$ & $\begin{array}{r}\text { Initial } \\
\text { value/mm }\end{array}$ & $\begin{array}{r}\text { Structural } \\
\text { parameter }\end{array}$ & $\begin{array}{r}\text { Initial } \\
\text { value/mm }\end{array}$ \\
\hline$D$ & 30 & $D_{1}$ & 26 \\
$D_{2}$ & 12 & $t_{\mathrm{p}}$ & 1.6 \\
$t_{\mathrm{m}}$ & 0.3 & $t_{\mathrm{c}}$ & 1.2 \\
\hline
\end{tabular}




\section{Optimization Method}

There are two optimization methods provided by ANSYS, i.e. the zero-order method and the first-order method. The zero-order method, due to its independency from using derivatives of the problem variables, is the first candidate for the optimization subroutine. It can be efficiently applied to most engineering problems (Zhang, Zhu, and Zhao 2010; Bang et al. 2008). The shape or material design optimization problem can generally be formulated as a constrained minimization problem as following (Zhang et al. 2012):

$$
\operatorname{Min} F=F(x)
$$

subjected to:

$$
g_{j}(x) \leq 0\left(j=1, \ldots, n_{\mathrm{c}}\right)
$$

with the design space:

$$
x_{i l} \leq x_{i} \leq x_{\text {iu }}(i=1, \ldots, N)
$$

where $F(\boldsymbol{x})$ is the objective function, $g_{j}(\boldsymbol{x})\left(j=1, \ldots, n_{\mathrm{c}}\right)$ are the constraint functions and $\boldsymbol{x}=\left[x_{1}, x_{2}, \ldots, x_{N}\right]$ is the vector of design variables. $x_{i l}$ and $x_{i u}$ describe physical upper and lower bounds on design variables. $n_{\mathrm{c}}$ and $N$ are the number of constraints and number of design variables, respectively. Solution of eqs [3]-[5] for shape optimization problems can be efficiently done by replacing objective and constraint functions with their response surface (RS) approximations. For this method, the dependent variables are replaced with the RS approximations by means of least squares fitting, and minimization is performed every iteration on the penalized function. The approximate optimization method implemented in ANSYS optimum module for the objective or constraint function as follows:

$$
\tilde{F}=a_{0}+\sum_{i=1}^{N} a_{i} x_{i}+\sum_{i=1}^{N} \sum_{j=1}^{N} b_{i j} x_{i} x_{j}
$$

where $a_{i}$ and $b_{i j}$ are coefficients to be determined. $\tilde{F}$ is the approximation of the objective function. For the zeroorder method, convergence is assumed if any one of the following conditions is satisfied:

$$
\begin{gathered}
\left|F^{(j)}-F^{(j-1)}\right| \leq \tau \\
\left|F^{(j)}-F^{(b)}\right| \leq \tau \\
\left|x_{i}^{(j)}-x_{i}^{(j-1)}\right| \leq \rho_{i}(i=1,2,3, \ldots, n) \\
\left|x_{i}^{(j)}-x_{i}^{(b)}\right| \leq \rho_{i}(i=1,2,3, \ldots, n)
\end{gathered}
$$

where $F^{(b)}$ is the best objective function at the current iteration. $\tau$ and $\rho_{i}$ are the objective function and design variable tolerances, respectively.

\section{Optimization Procedures}

\section{Objective Function}

Large vibration amplitude can improve the performance of the piezoelectric linear actuator. That is to say, it is needed to increase the vibration amplitudes of the cymbal stack transducer's axial stretching vibration. Arranging the saw electrical potential cycle by FFT idea, the mathematical model of optimization design can be expressed as follows:

$$
\left\{\begin{array}{l}
\text { Max. } U\left(P_{i}\right) \\
\text { s.t. } P_{i \min } \leq P_{i} \leq P_{i \max }
\end{array}\right.
$$

where $U$ is the top displacement of the cymbal stack transducer , $P_{i}$ is the structural parameters of the cymbal transducer, $P_{i \min }$ and $P_{i \max }$ are the minimum and maximum value of variable $P_{i}$, respectively.

The ANSYS program always minimizes the objective function, so the objective function is transformed to the following minimum problem:

$$
\text { Min. } F=C-U
$$

where $C$ is a constant, which is greater than the top displacement $U$.

\section{Design Variables}

The structure parameters of piezoceramic ( $D$ and $t_{\mathrm{p}}$ ) remain unchanged, because the limits of the piezoceramic processing technique and the design requirement. So, the other design parameters are chosen as design variables as follows:

$$
x=\left[\begin{array}{llll}
D_{1} & D_{2} & t_{\mathrm{m}} & t_{\mathrm{c}}
\end{array}\right]
$$

The variation ranges of the design variables as shown in Table 2 . The variation ranges of the design variables are determined according to the following reasons: the dimension constraint of different components of the piezoelectric linear actuator, and the previous experiences in cymbal transducer research.

\section{Optimization Process}

The optimization process of cymbal stack transducer design is shown in Figure 6. FEM is applied to the 
Table 2: Variation ranges of the design variables.

\begin{tabular}{lr}
\hline Design variable & Variation range $/ \mathrm{mm}$ \\
\hline$D_{1}$ & {$[18 ; 28]$} \\
$D_{2}$ & {$[8 ; 15]$} \\
$t_{\mathrm{m}}$ & {$[0.15 ; 0.5]$} \\
$t_{\mathrm{c}}$ & {$[0.8 ; 2]$} \\
\hline
\end{tabular}
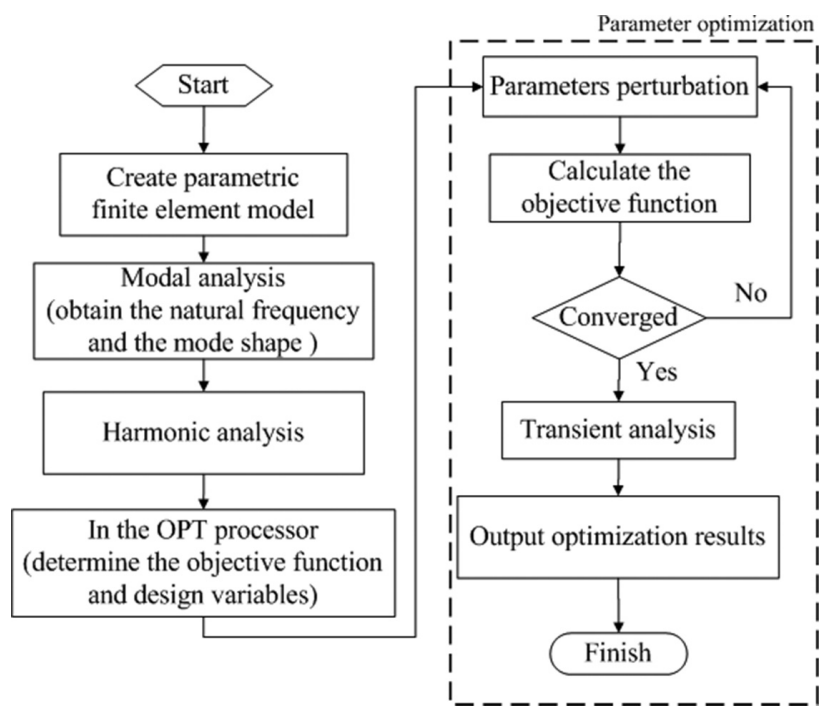

Figure 6: Flowchart of cymbal stack transducer.

modal analysis and harmonic analysis of the cymbal stack. Zero-order method is used to search optimum design of the design variables. The design process is programmed by ANSYS parametric design language (APDL). The iterative design process is as follows.

\section{Step 1: Parametric finite element model}

First, the input quantities such as dimensions, material properties and loads should be varied parametrically. So in the process of optimization, the three-dimensional model can be constructed in different dimensions. Meanwhile, according to the actual working conditions, the bottom surface of the cymbal stack transducer is clamped.

\section{Step 2: Modal analysis}

The modal analysis is used to determine the cymbal stack transducer's natural frequencies. Figure 7 shows the calculated mode shapes of the first four axial stretching vibrations. The first mode is known as the fundamental vibration mode of the cymbal stack transducer.

\section{Step 3: Harmonic analysis}

The frequency of the vibration modes depends on the geometry of the cymbal transducer. With the change of

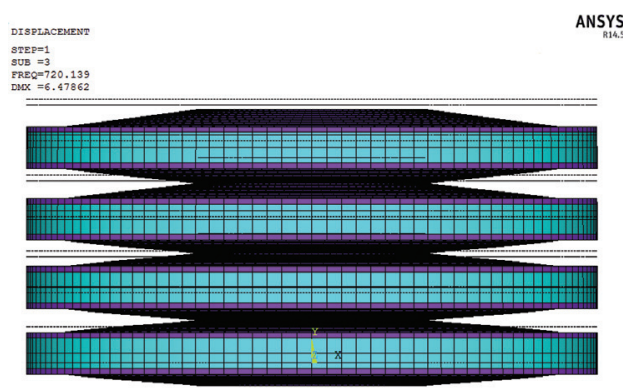

(a)

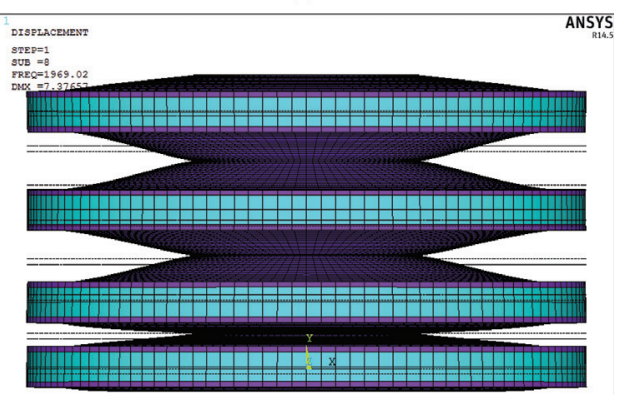

(b)

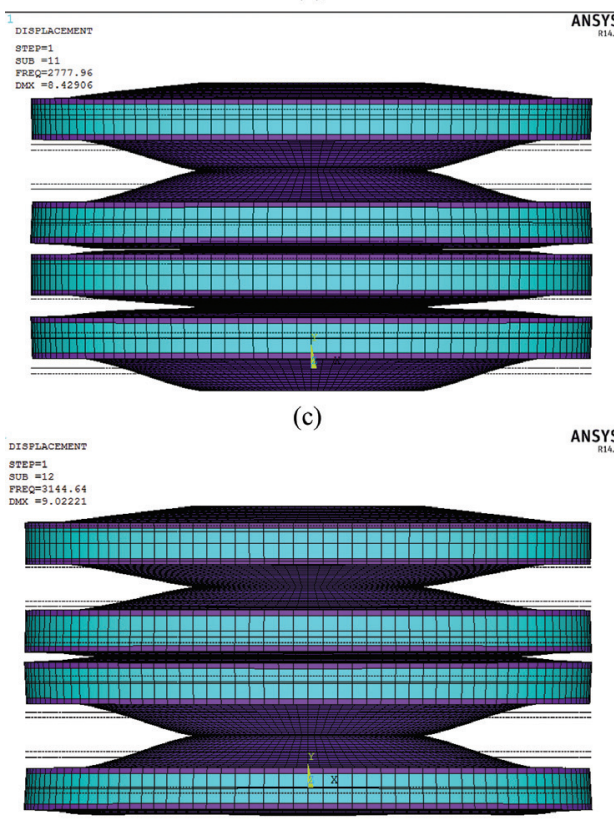

(d)

Figure 7: The axial stretching vibration mode shapes of the cymbal stack transducer: (a) the first-order mode shape at $720 \mathrm{~Hz}$, (b) the second-order mode shape at $1,969 \mathrm{~Hz}$, (c) the third-order mode shape at $2,778 \mathrm{~Hz}$ and (d) the fourth-order mode shape at 3,145 Hz .

the design variables, the frequency of the mode shape has also changed during the iterative process. In addition to the axial stretching vibration modes, the cymbal stack transducer has other torsional modes, as shown in Figure 8. Therefore, how to distinguish the axial stretching vibration modes from the other modes is one of the important problems to be solved. 

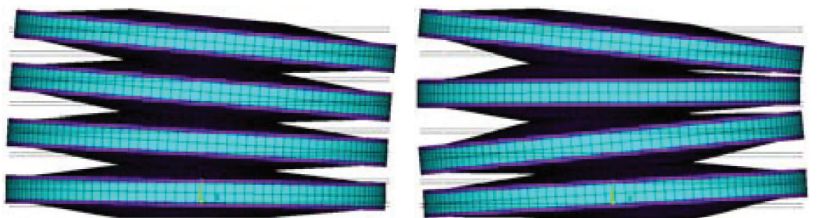

(a) $352 \mathrm{~Hz}$

(b) $1,101 \mathrm{~Hz}$

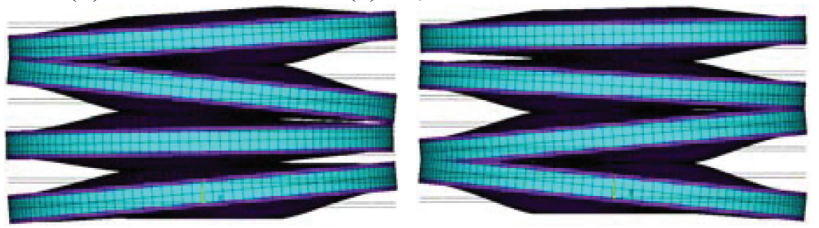

(c) $1,757 \mathrm{~Hz}$

(d) $2,132 \mathrm{~Hz}$

Figure 8: The other torsional vibration modes.

When the axial stretching vibration is generated, the displacement along the $Y$-axis of the top surface is much larger than the other modes, as shown in Figure 9. Thus, the desired axial stretching vibration can be obtained accurately by using the harmonic analysis.

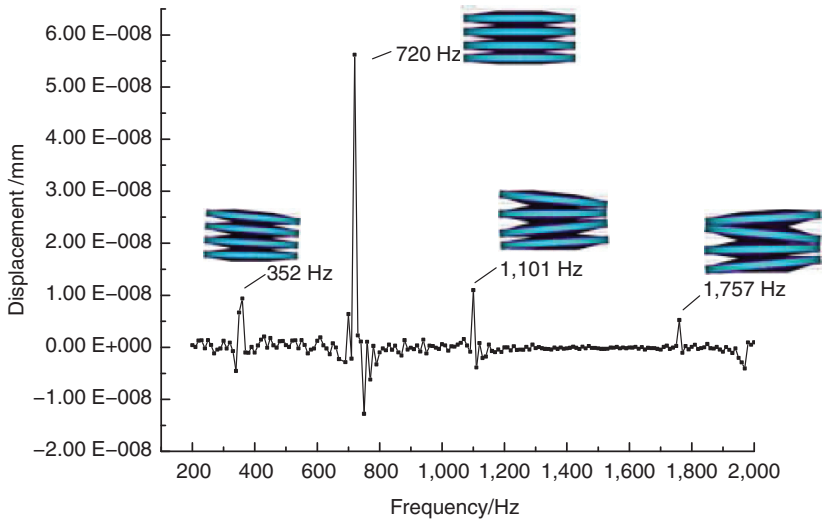

Figure 9: The diagram of harmonic analysis.

Step 4: Optimization history

The iterative history of the design variables is shown in Figure 10. After 24 iterations, the objective function and design variables reach a steady state and iteration termination. The design variables before and after the optimization are shown in Table 3.

Step 5: Transient analysis

Transient analysis is used to determine the dynamic response of the cymbal stack transducer under electric load. According to the Fourier analysis, a sawtoothshaped waveform can be decomposed into multiple sinusoidal waves:

$$
f(t)=A \sin (\omega t)+\frac{A}{2} \sin (2 \omega t)+\frac{A}{3} \sin (3 \omega t)+\cdots
$$

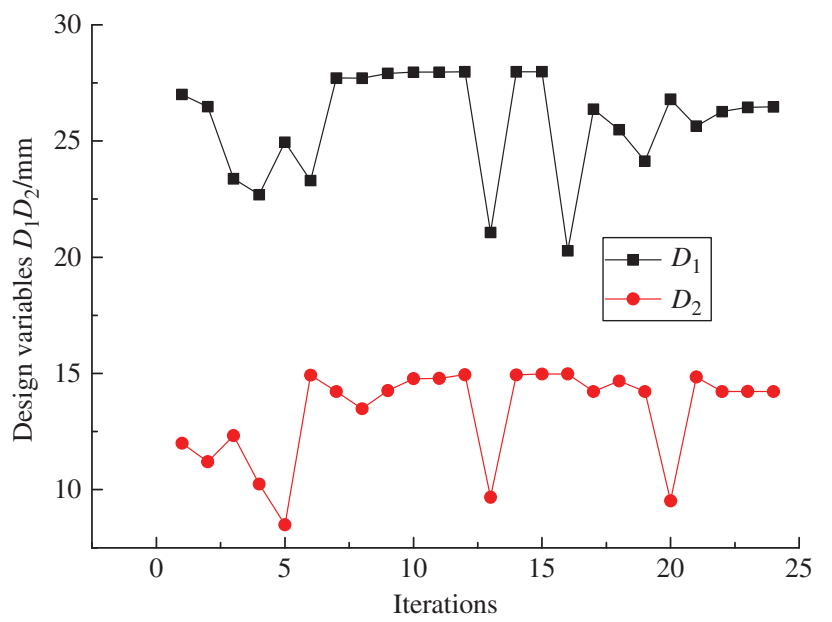

(a)

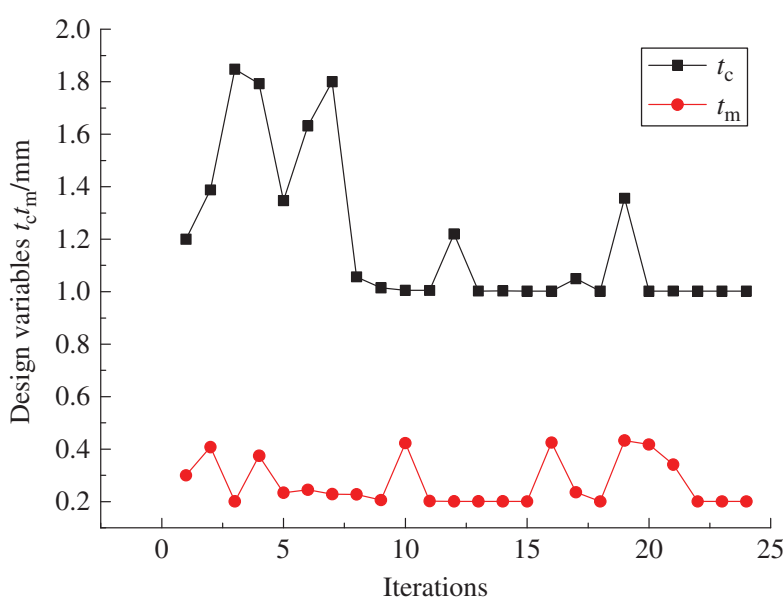

(b)

Figure 10: Iteration histories of design variables.

Table 3: Initial and final values of design variables and natural frequencies.

\begin{tabular}{llrr}
\hline & & $\begin{array}{r}\text { Initial } \\
\text { value }\end{array}$ & $\begin{array}{r}\text { Final } \\
\text { value }\end{array}$ \\
\hline Design variable & $D_{1} / \mathrm{mm}$ & 26 & 26.4 \\
& $D_{2} / \mathrm{mm}$ & 12 & 14.2 \\
& $t_{\mathrm{m}} / \mathrm{mm}$ & 0.3 & 0.24 \\
& $t_{\mathrm{c}} / \mathrm{mm}$ & 1.2 & 1 \\
Natural frequency of & First order/Hz & 720 & 683 \\
axial stretching & Second order $/ \mathrm{Hz}$ & 1,969 & 1,850 \\
vibration & Third order $/ \mathrm{Hz}$ & 2,778 & 2,581 \\
& Fourth order/Hz & 3,145 & 2,904 \\
\hline
\end{tabular}

where $f(t)$ is a sawtooth-shaped wave function, and $A$ is the amplitude of the first sinusoidal signal. A sawtooth-shaped waveform is composed of numerous integers multiple harmonics, the second harmonic is 
half the level of the first, the third harmonic is onethird the level of the first and so on. Because the coefficients of the third and higher harmonics are much smaller than the first two harmonics, the third and higher harmonics can be neglected in practice. To synthesize the axial stretching vibration, the ratio of the cymbal stack transducer's second and fundamental resonant frequencies can be designed to $4: 1$, as shown in Figure 11.

To verify the optimization results, the dynamic response of the cymbal stack transducer at the same

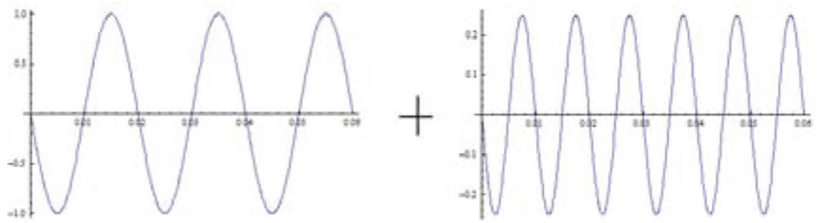

First res. frequency Second res. frequency

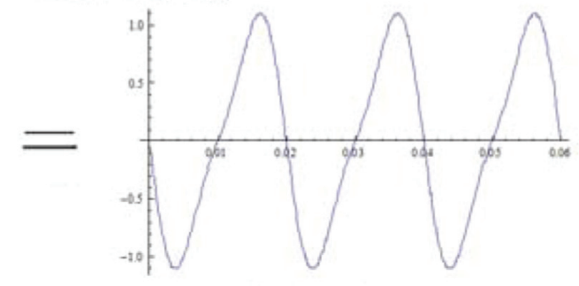

Amplitude ratio: $\quad 4: 1$

Figure 11: Two axial stretching vibration modes. voltage amplitude is numerically analyzed by using the transient analysis. The voltage amplitude applied on the surface of the piezoceramics is $200 \mathrm{~V}$. The top surface displacement curves of the cymbal stack transducer before and after optimization are shown in Figure 12. It can be seen from the figure that the maximum displacement generated by the cymbal stack transducer before optimization is $1.46 \mu \mathrm{m}$ and the maximum displacement is $1.94 \mu \mathrm{m}$ after optimization. The displacement value after optimization is increased by $32.9 \%$ compared with the value before optimization.

\section{Conclusion}

This research investigates on the conceptual design and performance analysis of a novel piezoelectric linear actuator. Based on the parametric finite element model, the optimization design of the cymbal stack transducer is realized by using the zero-order method. After optimization design, the displacement on the top surface is increased by $32.9 \%$ compared with the case of initial configuration. The parametric optimization method can quickly search for the optimal solution to meet the design requirements, which provides a new way for the optimization design of the piezoelectric linear actuator.

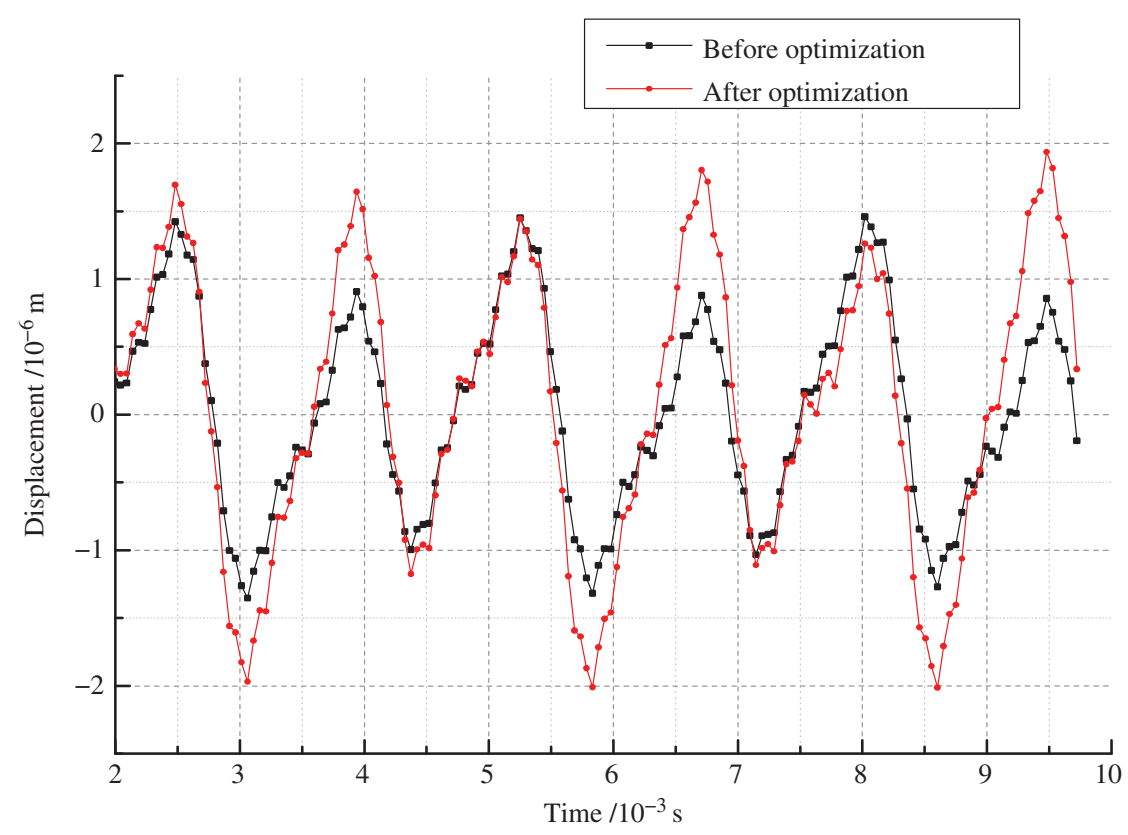

Figure 12: Displacement curve of cymbal stack transducer. 
Funding: This work was funded by National Natural Science Foundation of China (grant number: 51177053), Guangdong Province Science and Technology Innovation Project (grant number: 2012CXZD0016) and Specialized Research Fund for the Doctoral Program of Higher Education of China (grant number: 20124404110003).

\section{References}

Alibeigloo, A., M. Shakeri, and A. Morowa. 2007. "Optimal Stacking Sequence of Laminated Anisotropic Cylindrical Panel Using Genetic Algorithm." Structural Engineering and Mechanics 25 (6): 637-52.

Ameri, E., M. M. Aghdam, and M. Shakeri. 2012. "Global Optimization of Laminated Cylindrical Panels Based on Fundamental Natural Frequency." Composite Structures 94 (9): 2697-705.

Araujo, A. L., J. F. A. Madeira, C. M. M. Soares, et al. 2013. "Optimal Design for Active Damping in Sandwich Structures Using the Direct Multisearch Method." Composite Structures 105: 29-34.

Bajuri, M. N., M. R. A. Kadir, M. M. Raman, et al. 2012. “Mechanical and Functional Assessment of the Wrist Affected by Rheumatoid Arthritis: A Finite Element Analysis.” Medical Engineering \& Physics 34 (9): 1294-302.

Bang, I. K., D. S. Han, G. J. Han, et al. 2008. "Structural Optimization for a Jaw Using Iterative Kriging Metamodels." Journal of Mechanical Science and Technology 22 (9): 1651-9.

Chakraborti, N. 2004. "Genetic Algorithms in Materials Design and Processing." International Materials Reviews 49 (3-4): 246-60.

Chen, W. M., and T. S. Liu. 2013. "Modeling and Experiment of Three-Degree-of-Freedom Actuators Using Piezoelectric Buzzers." Smart Materials and Structures 22 (10): 105006.

Chen, H., W. Peng, R. Ge, et al. 2009. “Optimal Design of Composite Laminates for Minimizing Delamination Stresses by Particle Swarm Optimization Combined with FEM." Structural Engineering and Mechanics 31 (4): 407-21.

Duan, Z., and Q. Wang. 2005. "Development of a Novel High Precision Piezoelectric Linear Stepper Actuator." Sensors and Actuators A: Physical 118 (2): 285-91.

Hunstig, M., T. Hemsel, and W. Sextro. 2013. "Stick-Slip and SlipSlip Operation of Piezoelectric Inertia Drives. Part I: Ideal Excitation." Sensors and Actuators A: Physical 200: 90-100.

Jayachandran, K. P., J. M. Guedes, and H. C. Rodrigues. 2011. "Ferroelectric Materials for Piezoelectric Actuators by Optimal Design.” Acta Materialia 59 (10): 3770-8.
Kim, S. M., S. Wang, and M. J. Brennan. 2011. "Dynamic Analysis and Optimal Design of a Passive and an Active Piezo-electrical Dynamic Vibration Absorber." Journal of Sound and Vibration 330 (4): 603-14.

Kulkarni, R. V., and G. K. Venayagamoorthy. 2011. "Particle Swarm Optimization in Wireless-Sensor Networks: A Brief Survey." IEEE Transactions on Systems, Man, and Cybernetics, Part C: Applications and Reviews 41 (2): 262-7.

Lee, J., W. S. Kwon, K. S. Kim, et al. 2011. "A Novel Smooth Impact Drive Mechanism Actuation Method with Dual-Slider for a Compact Zoom Lens System." Review of Scientific Instruments 82 (8): 085105.

Liu, Y., J. Liu, W. Chen, et al. 2010. "A Cylindrical Traveling Wave Ultrasonic Motor Using Longitudinal Vibration Transducers." Ferroelectrics 409 (1): 117-27.

Lu, H., J. Zhu, Z. Lin, et al. 2009. "An Inchworm Mobile Robot Using Electromagnetic Linear Actuator.” Mechatronics 19 (7): 1116-25.

Morita, T., H. Murakami, T. Yokose, et al. 2012. "A Miniaturized Resonant-Type Smooth Impact Drive Mechanism Actuator." Sensors and Actuators A: Physical 178: 188-92.

Narayanan, M., and R. W. Schwartz. 2007. "Finite Element Modeling of a Donut Flextensional Transducer." Journal of the American Ceramic Society 90 (3): 850-7.

Park, J., S. Lee, and B. M. Kwak. 2012. "Design Optimization of Piezoelectric Energy Harvester Subject to Tip Excitation.” Journal of Mechanical Science and Technology 26 (1): 137-43.

Poli, R., J. Kennedy, and T. Blackwell. 2007. "Particle Swarm Optimization." Swarm Intelligence 1 (1): 33-57.

Szufnarowski, F., and A. Schneider. 2011. "Force Control of a Piezoelectric Actuator Based on a Statistical System Model and Dynamic Compensation." Mechanism and Machine Theory 46 (10): 1507-21.

Tsivgouli, A. J., M. A. Tsili, A. G. Kladas, et al. 2007. "Geometry Optimization of Electric Shielding in Power Transformers Based on Finite Element Method." Journal of Materials Processing Technology 181 (1): 159-64.

Watson, B., J. Friend, and L. Yeo. 2009. "Piezoelectric Ultrasonic Micro/Milli-scale Actuators." Sensors and Actuators A: Physical 152 (2): 219-33.

Zhang, J., H. Zhu, and C. Zhao. 2010. "Combined Finite Element Analysis and Subproblem Approximation Method for the Design of Ultrasonic Motors." Sensors and Actuators A: Physical 163 (2): 510-15.

Zhang, J. T., H. Zhu, S. Q. Zhou, et al. 2012. “Optimal Design of a Rod Shape Ultrasonic Motor Using Sequential Quadratic Programming and Finite Element Method." Finite Elements in Analysis and Design 59: 11-17. 